\begin{tabular}{|c|c|c|c|}
\hline NATAL & $\begin{array}{l}8^{\text {th }} \text { CIDI } \\
8^{\text {th }} \text { Information Design } \\
\text { International Conference }\end{array}$ & $\begin{array}{l}8^{\text {th }} \text { CONGIC } \\
8^{\text {th }} \text { Information Design } \\
\text { Student Conference }\end{array}$ & $\begin{array}{l}\text { Blucher Design Proceedings } \\
\text { Junho } 2018 \text {, num. } 1 \text {, vol. } 4 \\
\text { proceedings.blucher.com.br }\end{array}$ \\
\hline
\end{tabular}

\title{
Proposta de modelo descritivo para o estilo de ilustração em Sequência Pictórica de Procedimento em bulas de medicamentos
}

\author{
Proposal of a descriptive model for the illustration style in Procedural Pictorial \\ Sequence in medicine leaflets.
}

Luiz Gustavo Amorim Lopes; Carla Galvão Spinillo

\begin{abstract}
Estilo de ilustração, Bula de medicamentos, Sequência Pictórica de Procedimento
O propósito deste artigo é verificar o estilo de ilustração em sequência pictórica de procedimento, cujo objetivo se delimita em realizar um modelo descritivo para o estilo de ilustração em bulas de medicamentos. O método utilizado para compor esse estudo, consiste na validação do modelo junto aos participantes - distribuídos entre desenvolvedores e especialistas - para a validação do modelo descritivo, elaborado com base em dois modelos avaliativos: Ashwin (1979), destinado às variáveis do estilo de ilustração, e, Spinillo (2001), para a composição dos elementos de representação contidos nas instruções visuais. Este artigo está estruturado da seguinte maneira: (1) Introdução; (2) Estilo de ilustração apresentando os principais conceitos e classificações do estilo visual; (3) Sequência pictórica de procedimentos (SPPs) em bulas de medicamentos; (4) Método, com o detalhamento da elaboração dos modelos e como utilizá-lo para a descrição das SPPs em bulas de medicamentos, (5) Resultados levantados junto aos participantes com o desempenho do modelo para descrição do estilo presente nas bulas de medicamentos; (6) Considerações finais - apresentando as principais modificações e ajustes ao modelo descritivo para o estilo.
\end{abstract}

Illustration Style, Medication Leafet, Procedural Pictorial Sequence

The purpose of this paper is to evaluate the instructional illustration style in Procedural Pictorial Sequence in medicinal package inserts. The purpose delimit on model descriptive in the illustration style. The method applied in this study consists on validation of the model with the participants - distributed among developers and experts - for the validation of the descriptive model, based in information obtained from two evaluation models: Ashwin (1979), for the style of variables Illustration, and, Spinillo (2001), about the composition of the elements of representation contained in the visual instructions. This paper is structured as follows: (1) Introduction; (2) illustration style - presenting the main concepts and classifications of the visual style; (3) Procedural Pictorial Sequence (PPS) in medicinal leaflets; (4) Method, with details of the elaboration of the model and how to use it on the description of PPSs in medicinal leaflets, (5) Results obtained from the participants about the performance of the model on style medication leafet; (6) Final considerations - presenting the main modifications and adjustments to the descriptive model for the style.

\section{Introdução}

As comunicações por meio da imagem são recurso recorrente nos dias de hoje, contudo ressaltamos para a importância das instruções visuais. Redig (2004) lembra da ocorrência de um acidente com um ônibus de transporte público incendiado e que resultou na morte de 10 passageiros, o que poderia ter sido evitado, uma vez que veículo possuía saídas de emergência. $O$ autor questiona se as pessoas não conseguiram compreender as instruções de funcionamento das saídas de emergência ou se não conseguiram operá-las por terem sido mal planejadas, em ambos os casos, apontando para problemas relacionados ao design: da informação ou industrial. Para não estar relacionado ao design, o que poderia ter acontecido na ocasião era o mecanismo de abertura ter apresentado problemas.

Anais do 8을 e 8 CONGIC

Guilherme Santa Rosa; Cristina Portugal (orgs.)

Sociedade Brasileira de Design da Informação - SBDI

Natal | Brasil | 2017

ISBN 978-85-212-1305-5
Proceedings of the $8^{\text {th }}$ CIDI and $8^{\text {th }}$ CONGIC

Guilherme Santa Rosa; Cristina Portugal (orgs.)

Sociedade Brasileira de Design da Informação - SBDI

Natal| Brazil | 2017

ISBN 978-85-212-1305-5 
Assim, imagens, muitas vezes utilizadas como instrumentos facilitadores da comunicação, podem ser ineficientes para transmitir uma mensagem, causando problemas em relação à compreensão da informação que se almeja transmitir, destacamos para a área da saúde em especial, as ilustrações das bulas de medicamentos destinados aos usuários e/ou pacientes.

As normativas para as bulas de medicamentos são regulamentadas pela Agência Nacional de Vigilância Sanitária (ANVISA), que estabelece a Resolução da Diretoria Colegiada (RDC) n 140 de 2003 e RDC n 47 de 2009, para esse tipo de documento, contudo não especificam as características para a composição e a elaboração das ilustrações instrucionais.

Entretanto, o estado do Paraná desenvolveu uma legislação exclusiva às farmácias de manipulação, com a obrigatoriedade no fornecimento das bulas para medicamentos magistrais $^{1}$ (PARANÁ, 2013a). A resolução Secretária do Estado da Saúde (SESA) no 62 de 2013, estabelece algumas normativas com relação à construção desse tipo de documento além das características textuais (fonte, tamanho e espaçamento) que também é abordado pela RDC ํㅜ 140. Contudo apenas a SESA no 62 faz algumas recomendações para as ilustrações procedimentais, como forma de representar movimento, através do uso de setas, evitar o excesso de detalhes na representação dificultando seu entendimento e devem ser constituídas com fundo neutro (PARANÁ, 2013b).

Apesar disso a variação destas características de uma ilustração se dá pelo seu Estilo empregado, podendo comprometer a percepção e a compreensão da imagem. Para Gombrich (1995) o Estilo é o fator que diferencia uma representação, seja por meio da aplicação de diferentes técnicas ou pelas características da própria personalidade do ilustrador.

Outro ponto é que as ilustrações instrucionais, não possuem normativa para a sua concepção, ou seja, os laboratórios passam a desenvolver de maneira livre, por não possuir critérios normativos para os ilustradores e/ou desenvolvedores de bulas de medicamentos, além de não considerarem outros fatores como: o entendimento do usuário/paciente com relação ao estilo; a legibilidade das imagens representadas e a compreensão do conteúdo informado de forma visual por meio das ilustrações procedimentais.

Os aspectos acima mencionados levam a constatação da necessidade de uma linguagem visual eficiente, por meio de ilustrações em bula de medicamentos, atendendo às condições de entendimento da mensagem pelo público-alvo, assim o estilo visual pode ser o fator determinante, em que muitas vezes não é obsevado pelos desenvolvedores nesse tipo de material, ou pelos laboratórios de medicamentos, já que a forma de representar não existe critérios ou normativas, em relação ao estilo de ilustração aplicada às bulas de medicamentos.

\section{Estilo de ilustração}

A representação visual é algo analisado e questionado por diversas gerações e por longos anos se reflete a mesma indagação, como as pessoas "representam o mundo visível de maneiras tão diferentes? ", Estabelecendo como o fator principal para se realizar essa diferenciação das representações do mundo o Estilo (GOMBRICH, 1995, p.3).

Assim o significado para o estilo, está diretamente relacionado à etimologia da palavra, cuja origem grega, stylo, indicando coluna e no latim, stilus, significando uma haste de planta ou ferro de ponta, utensílio utilizado para escrever em tábuas de madeira revestidas de cera, semelhante a um caderno, onde se faziam anotações cotidianas, com possibilidade de serem apagadas ou alteradas por meio da raspagem da cera, substituída, depois, por nova camada (ANZOLCH, 2009).

Para Gombrich (1995) determina o estilo como as características presentes sejam por meio de uma escola de artes ou do método de descrição que tem como objetivo:

\footnotetext{
${ }^{1}$ As abrangências desta resolução são os estabelecimentos farmacêuticos - farmácias que manipulam medicamentos para uso humano (PARANÁ, 2013b, p. 1).
} 
agrupar, organizar e identificar, diferentes técnicas utilizadas para se realizar a distinção de um estilo para o outro, compreendendo como as mais diversas ideias desde temperamento e/ou personalidade, preferências pessoal e/ou artística, movimento artístico, escola de arte ou períodos históricos.

O Campo visual para Dondis (1997, p. 161) determina o estilo como uma "síntese visual de elementos e técnicas, sintaxe, inspiração, expressão e finalidade básica" como a melhor maneira de distingui-lo é perceber como a ordem e as categorias de expressão visual se apresentam. Assim os códigos estilísticos, se configuram pelos mínimos detalhes ao invés de apenas convenções para o controle, classificando as variações de técnicas, como forma de identificar a sua individualidade.

O estilo delimita como uma qualidade que possibilita a distinção do individuo ou grupo de pessoas, do mesmo modo que a linguagem pictórica o estilo aplica-se a todos os meios de produção incluindo a fotografia, entretanto o estilo pode atribuir-se as mais variadas formas e recursos, a sua disposição (e.g. vertical, horizontal) a relação espacial, contraste de tons, entre outras maneiras distintas, concluindo como não sendo uma tarefa fácil a sua descrição em palavras (TWYMAN, 1985).

Ashwin (1979) trata o estilo em relação à ilustração contemporânea, com a identificação das variáveis, com base na sua presença ou ausências de tais fatores. Contudo vale resaltar que o autor não relaciona a palavra estilo a imagem, mas suas características estilísticas relacionadas aos períodos históricos ou artísticos (e.g clássico impressionista, surrealista). Observa que o estilo na história da arte busca ser "retrospectiva do que pragmática, preocupando-se com a mudança de época, cíclica ou evolutiva" (ASHWIN, 1979, p.51).

\subsection{Classificação do estilo visual}

A classificação do estilo pode ocorrer em diferentes formas e critérios a sua inclusão ou não a um determinado estilo de representação. Dondis (1997) classifica com base nos métodos e técnicas, como uma categoria de elementos diferenciadores, além do fator do estilo individual ou em grupo, a influência do ambiente social, físico, político e psicológico são características fundamentais para o que se produz ou expressa de forma visual, assim a sua classificação para representar a imagem, distribui-se em cinco grandes grupos:

1. Primitivo: encontrados nas primeiras pinturas rupestres, configurado pelo uso de técnicas bem simples, mas com uma riqueza de símbolos constantemente encontrados na escrita, inspirados na natureza, busca realizar com o máximo de realismo seus desenhos, atribuindo significados e tornando o aprendizado uma fonte de informação, as técnicas abordadas buscam a intensificar o significado da mensagem por meio da simplicidade dos elementos presentes nas ilustrações;

2. Expressionista: semelhante à primeira categoria por ambas realizam o exagero da ilustração, esta se distingui por distorcer a realidade representada, o expressionismo faz a abstração do real juntos aos sentimentos, alcançando resultados mais emocionais;

3. Clássico: típicos da arte grega diferenciam essas categorias do expressionismo por contrastar com a racionalidade, consequentemente abordado por meio de formulas ou proporção áurea, configurado pela sua aproximação com a realidade em suas obras por meio de técnicas de perspectivas e tratamento da luz a fim de alcançar o realismo ao extremo;

4. Ornamental: caracterizado como a categoria puramente decorativa, configuradas por elementos mais acentuados, com estruturas em ângulos mais agudos de modo a transmitir habilidades e elegância nas representações associando a riqueza e poder.

5. Funcional: considerado como a categoria mais contemporânea classificada pela sua funcionalidade, categoria derivado da revolução industrial, buscando propor soluções para problemas básicos do dia-a-dia, mas que não se ausentassem das qualidades estéticas.

\section{A classificação proposta por Dondis (1997) mesmo apresentando em mais de uma}


categoria do estilo, não se aprofunda em relação à variação, apenas limitando as técnicas empregadas, movimento artísticos e/ou períodos históricos.

Para McCloud (1995, p. 27) fundamentados no vocabulário dos quadrinhos, classifica o ícone como "qualquer imagem que represente uma pessoa ou algo", distribuídos em três formas: ícones simbólicos utilizados para a demonstração de conceitos e/ou ideias; ícones de linguagem configurando como a escrita ou a mensagem verbal para 0 seu entendimento; ícone figura como imagens que se assemelham ao conteúdo, algumas, entretanto mais abstratas do que as outras.

De tal forma, os significados dos ícones simbólicos são fixos, representando "ideias invisíveis", os ícones de linguagem são letras totalmente abstratas, se não estiverem agrupadas formando uma palavra e, logo, um significado, comparadas com os ícones de figura, com a representação das suas variáveis, que remetem a diferentes níveis, inclusive à abstração da sua forma. (MCCLOUD, 1995).

A classificação McCloud (1995) limita-se ao significado da imagem, diferentemente da proposta por Dondis (1997), que faz a classificação do estilo pelas técnicas empregadas. Para McCloud (1995) demonstra diferente abordagem por meio da abstração da imagem real, demonstrando a capacidade de se reter informação por meio da simplificação da sua representação, técnicas muito utilizadas nos quadrinhos, a fim de alcançar o maior número de pessoas o seu significado.

Entretanto para os objetos (e.g. cadeira, livro, carro), realiza a aproximação com a imagem real, assim para as histórias em quadrinhos a junção de dois estilos de representação na mesma cena, contudo vale resaltar que a abstração em alguns objetos pode dificultar o entendimento, mas para outros, aproximar do leitor (MCCLOUD, 1995).

Diferentes dessas abordagens Ashwin (1979), acredita que a decisão para formação da representação, são realizadas pelo próprio ilustrador, sendo o mesmo responsável pelas características que são atribuídas ao desenho, destacando para que as decisões consciente ou inconsciente, ocorram de forma espontânea, a fim de compor a avaliação do ilustrador e da ilustração.

Ashwin (1979) classifica as variações do estilo de ilustração por meio de polos opostos, de acordo com o seu detalhamento, está classificação busca verificar as características estilísticas no campo semântico da imagem do qual é composto por sete elementos do estilo (Quadro 1): consistência, gama, enquadramento, posicionamento, proxêmica, cinética e naturalismo. Uma imagem, seja ela uma fotografia ou uma ilustração, posiciona-se nos extremos dos polos a uma ou mais variáveis, que determinam o caráter estilístico de uma imagem. (ASHWIN, 1979).

Quadro 1 - Variável do estilo visual - Fonte: ASHWIN, 1979, p. 57.

\begin{tabular}{l|l|l}
\cline { 2 - 3 } & POLOS & \\
\hline Consistência & Homogênea & Heterogênea \\
\hline Gama & Expandida & Restritiva \\
\hline Enquadramento & Conjuntiva & Disjuntivo \\
\hline Posicionamento & Simétrico & Casual \\
\hline Proxêmica & Perto & Distante \\
\hline Cinética & Dinâmico & Estático \\
\hline Naturalismo & Naturalista & Não-Naturalista \\
\hline
\end{tabular}

- Consistência: configurada pelas características da imagem, (e.g. linhas, espessura, peso, direção) seus polos se posiciona entre a homogênea configurando como uma unidade na representação e heterogênea não configurando uma coerência visual da imagem; 
- Gama: são as possibilidades sintáticas da imagem, enquadra as diferentes possibilidades sintáticas, de modo que a escolha do ilustrador desenvolve as limitações autoimpostas, proporcionando uma gama de possibilidades para a imagem (e.g. direção e comprimento da linha, forma, escala do objeto, limitação de gama de cores ou combinações, intensidade dos efeitos). A variação dos polos posiciona-se com a sua restrição ou a sua expansão.

- Enquadramento: é como os elementos estão apresentados e dispostos na imagem, variando entre conjuntiva em que o seu significado se expande as todas as dimensões descentralizando a sua atenção, criando a percepção de profusão e diversidade. Entretanto a abordagem disjuntiva faz o contrário realizando a separação da relação ao motivo e ambiente, a fim de prender a atenção do leitor por meio do foco em algum objeto ou ação representada.

- Posicionamento: consiste na composição da imagem em relação aos seus elementos que são distribuídos na cena, pode ser casual por não priorizando a importância em um único elemento, mas em todo o seu plano, e para a categoria simétrica também associada a categoria disjuntivo consistindo no seu foco em um único elemento a ser represtado ou a configurar atenção do leitor.

- Proxêmica: constitui na representação implícita do espectador com o leitor, os seus polos fazem o posicionamento (perto/distante) por meio da variação da escala da figura.

- Cinética: variável responsável pela representação de movimento através de aspectos delimitados pelo próprio ilustrador reforçando ou diminuindo no objeto por meio das linhas e/ou traços.

- Naturalismo: está última categoria se constrói com um conjunto das demais variáveis, alterando entre naturalista e não-naturalista, que se faz a seu posicionamento através da aproximação com o objeto real representado.

A classificação aborda por Ashwin (1979), destaca-se por detalhar a composição da ilustração diferenciando da abordagem realizada por McCloud (1995), que consiste na simplicidade da forma representada. Entretanto outra maneira de classificar o estilo visual por meio da variação visual da representação (FUSSELL \& HAALAND, 1978; COOK, 1980; DWYER \& PARKHURST, 1982; SPINILLO, 2001).

Fussell e Haaland (1979) destacam que desenhos estilizados configurados como simples, não há nada em sua representação, exigindo muito mais para se interpretar a imagem e compreendê-la. Para Cook (1980) as ilustrações em outline, (mínimo de detalhes) são que possuem as maiores dificuldades, considerando a representação figura vara ou boneco de palito como o estilo de menor preferência entre os participantes que se aplicou o seu estudo.

Spinillo (2001) define para Sequência Pictórica de Procedimento (SPP) como a representação procedimental por meio de uma série de passos, prioritariamente através das ilustrações, de modo a diferenciar do termo instruções visuais configurando como mais abrangentes. Assim Spinillo (2010) faz a delimitação de quatro grupos distintos para aplicar nas SPPs, compondo por:

- Estilo fotográfico $(\mathrm{A})$ : representação que possui baixo contraste tonal e alto grau de naturalismo.

- Estilo de desenho (B): representação que está em uma categoria mediana entre o estilo fotográfico e o esquemático.

- Estilo esquemático (C): ilustração que possui baixo grau de naturalismo devido à ausência de elementos e grau médio de contraste tonal.

- Estilo de sombra (D): ilustração semelhante ao do estilo esquemático em relação ao baixo grau de naturalismo, mas com nível alto de contraste tonal. Igual à silhueta, optase por esta nomenclatura por constituir um termo mais genérico, podendo, assim, incluir uma série maior de ilustrações. 
Figura 1 - Estilos de ilustração em Sequências instrucionais de procedimentos. Fonte: LA ROCCA, Naomi. In: SPINILLO, 2010, p. 26.

A

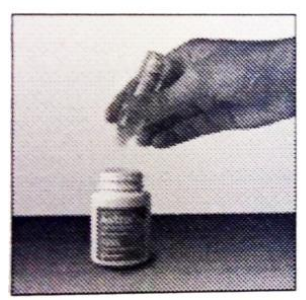

B

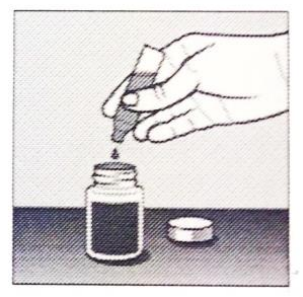

C

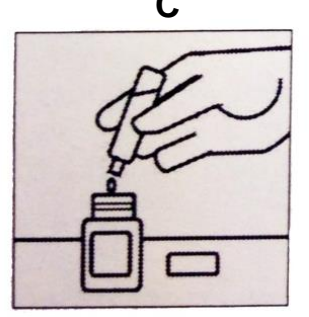

D

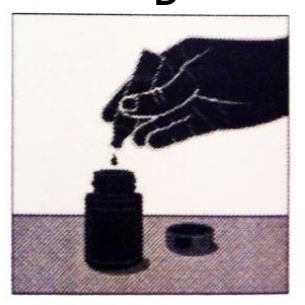

Portanto a classificação do estilo visual é abordada por diversos autores de diferentes maneiras esse assunto, destacando entre eles: os períodos histórico ou artístico por meio dos elementos de época, assim como as técnicas empregadas na construção da ilustração e as suas características (DONDIS, 1997); a abstração da imagem por meio da ausência de seus elementos (MCCLOUD, 1995); a variação visual, de modo que possa distinguir a figura através da sua diferenciação (FUSSELL \& HAALAND, 1978; COOK, 1980; DWYER \& PARKHURST, 1982; SPINILLO, 2001); e por fim através do detalhamento da imagem, com a categorização no campo semântico com a identificação dos seus elementos (ASWHIN, 1979).

\section{Sequências Pictórica de Procedimentos em Bulas de medicamentos}

A área da saúde, em especial a farmacoterapia, é alvo de constantes pesquisas no design (FUJITA \& SPINILLO, 2008; SPINILLO et al. 2011; FUJITA \& MACHADO, 2014; HAMMERSCHMIDT \& SPINILLO, 2015; HARADA \& SCHOR, 2016. DA SILVA \& SPINILLO, 2016), contudo, o material mais frequentemente observado e analisado pelos designers esta relacionado ao documento da bula de medicamento, cujo objetivo é realizar a comunicação com usuário/paciente.

A legislação vigente no país para os medicamentos fabricados nacionalmente ou importados, destacam a necessidade de informações em formato de documentos (Bulas de medicamentos) presente no interior de seus medicamentos (GONCALVES et. al. 2002). Assim sendo, a bula passa a ser um importante documento de consulta pública, pois fornece informações especificas sobre: composição química, precações, advertência, cuidados, preparo e até a forma de utilizar a medicação (FUJITA; SPINILLO, 2008).

Deste modo as instruções visuais em bulas de medicamentos possuem um papel fundamental para o sucesso da informação transmitida, já que alguns conteúdos são melhores representados na forma pictórica (e.g. tamanho relativo, conexão de partes), assim sendo, as ilustrações contribuem para o entendimento da mensagem textual, surgindo como um reforço ou complemento a informação (SPINILLO et. al. 2007).

A informação relacionada aos medicamentos não se limita apenas a sua disponibilidade, mas são organizadas de forma acessível e compreensível por todos que necessitam, independentemente de sua escolaridade, classe social ou situação em que se encontra. Essas características são importantes, e, devem ser destacadas, durante a construção de um projeto de design da informação nessa área (SCARIOTI; DE ARAUJO GOMES, 2009).

Para Spinillo (2001), o processo de elaboração de uma SPP é importante resaltar que ocorre como uma via de entrada única, com os produtores de um lado e os receptores do outro, não configurando como um processo de design linear em seu desenvolvimento, mas a integração de todas as partes envolvidas para a concepção da sequência procedimental. Ocorrendo da mesma maneira para as SPPs destinadas as bulas de medicamentos.

Algumas pesquisas relacionadas à Sequência Pictórica de Procedimento estabelecem a avaliação da análise da tarefa (SPINILLO et. al. 2008; 2011; SPINILLO \& WAARDE, 2011), e, limitam-se apenas a compreensão do seu conteúdo, não avaliando ou descrevendo o estilo de ilustração presente nesse tipo de documento. 


\section{CIDI 2017

\section{1 Modelo de análise em SPPs}

Para a análise e discrição em uma SPP, utiliza-se o modelo desenvolvido por Spinillo (2001) como o mais completo para verificar a representação dos passos de uma determinada atividade e/ou ação, por meio da ilustração a fim de fazer um levantamento dos elementos com maior incidência na representação. O modelo de análise das SPPs (Quadro 2) divide-se em oito categorias: apresentação do texto, disposição da sequência, orientadores de leitura, elementos de separação visual, elementos simbólicos, elementos enfáticos, estilo de ilustração e representação da figura.

Quadro 2 - Modelo de análise de SPP - Fonte: SPINILLO, 2001, p. 40.

\begin{tabular}{l|l}
\hline \multirow{2}{*}{ Apresentação do texto } & Legenda \\
& Texto corrido \\
& Rotulo \\
\hline Disposição da sequência & Horizontal \\
& Vertical \\
& Obliqua \\
& Circular \\
Ramificação \\
\hline Orientadores de leitura & Letras \\
\hline \multirow{2}{*}{ Elemento de separação visual } & Números \\
& Setas \\
\hline Elementos simbólicos & Bordas \\
& Linhaşos \\
\hline \multirow{2}{*}{ Elementos enfáticos } & Barra diagonal \\
& Setas \\
\hline & Cores \\
& Formas \\
\hline & Fotográficos \\
Desenho \\
Esquemático de ilustração & Sombra \\
\hline & llustração Parcial \\
& llustração completa \\
\hline
\end{tabular}

- Apresentação do texto: relação que se faz do texto à ilustração, maneira de disponibilizar o texto: legenda, quando a ilustração se encontra no interior do texto; rótulo, texto é inserido na ilustração; texto corrido.

- Disposição da sequência: as ilustrações podem ser organizadas de forma horizontal, vertical, oblíqua, ramificada ou circular.

- Orientadores de leitura: são caracterizados pelos elementos que auxiliam na leitura da sequência, como números e letras.

- Elementos de separação visual: elementos gráficos que conduzem a separação da ilustração na SPP, como bordas, espaços ou linhas.

- Elementos simbólicos: linguagem de convenção utilizada na representação, como seta para direção ou barra diagonal para caracterizar negação.

- Elementos enfáticos: meios utilizados para chamar a atenção do leitor para a SPP ou realçar detalhes na ilustração, com as variações de cores e formas. 


\section{CIDI 2017

- Estilos de ilustração: variações empregadas para representar a SPP com relação à técnica utilizada para compor a ilustração: esquemático, desenho, fotográfico e sombra.

- Representação da figura: forma de composição da ilustração, classificada como completa e incompleta, ou seja, parcial.

\section{Método}

A metodologia utilizada para se realizar esse levantamento, consiste na validação do modelo descritivo para estilo de ilustração em bulas de medicamentos. Assim essa abordagem pode ser assimilada ao estudo de campo, em que se faz a investigação direta dos fatos e fenômenos, em que a coleta dos dados ocorre o controle das variáveis relevantes para análise (MARCONI \& LAKATOS, 1996).

A validação do modelo descritivo utilizado nesse estudo configura-se em três etapas distintas: (1) Amostra das ilustrações instrucionais em bulas de medicamentos; (2) desenvolvimento do Modelo descritivo para estilo de ilustração; (3) Aplicação do modelo com os participantes. A seguir detalharemos as respectivas etapas que configuraram a validação do modelo descritivo para o estilo.

\subsection{Amostra das ilustrações instrucionais em bulas de medicamentos}

A composição da amostra limitou-se apenas as bulas digitais, disponíveis pelo site do bulário eletrônico da ANVISA, em que estão cadastradas todas as bulas comercializadas nacionalmente, possibilitando o acesso livre para toda a população brasileira. As bulas cadastradas estão configuradas no formato Portable Document Format (PDF) em que o sistema disponibiliza a leitura online, assim como a possibilidade de realizar a impressão do documento no formato A4 $(21 \times 29,7 \mathrm{~cm})$.

Para a composição da amostra a ser aplicado aos participantes, de modo que se possa validar o modelo descritivo, foi necessário preservar o mesmo formato que estão cadastradas as bulas digitais anexadas no bulário eletrônico. Contudo de modo a delimitar as ilustrações optou-se pela ausência dos seus textos, afim de não influenciar na descrição do estilo da ilustração pelos participantes.

\subsection{Desenvolvimento do modelo descritivo para estilo de ilustração}

A condução para o desenvolvimento do modelo descritivo para estilo de ilustração em SPPs utilizou-se como base para a sua concepção os modelos de Spinillo (2001) e Ashwin (1979). Observando esses exemplos, optou em elaborar uma ferramenta descritiva abordando dois pontos principais, composto por:

1. Variáveis do estilo: consiste nos elementos de concepção da ilustração, a qual se relaciona com a escolha da forma e técnicas utilizadas para a representação instrucional.

2. Elementos de representação na SPP: compostas por todos os elementos presente na representação de uma SPPs, ou seja, não se enquadrando como um estilo de ilustração, configurando como um acréscimo e/ou acessórios à representação.

Os resultados provenientes dessas categorizações desenvolveram-se duas versões o modelo descritivo, a primeira com a utilização da escala Likert para descrição das SPPs ao todo, e para segunda versão, aplicou-se a descrição em quadros separados para as ilustrações, como forma de melhor detalhar o estilo empregado nas ilustrações procedimentais. Entretanto, observou a necessidade de realizar testes com as versões dos modelos desenvolvidos, a fim de verificar o desempenho para a função de descrever o estilo de ilustração. 


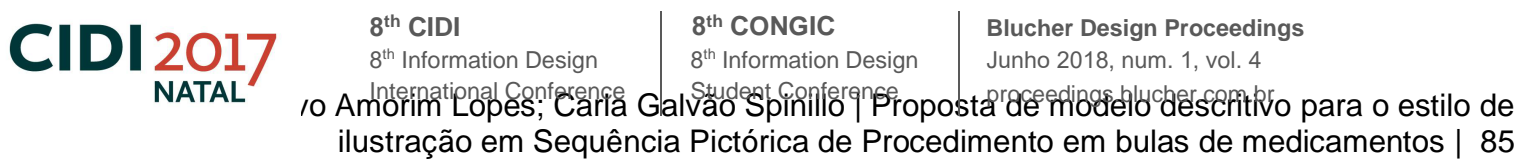

\subsection{Aplicação do modelo com os participantes}

Os participantes selecionados para compor o estudo de validação são compostos por ambos os sexos e com faixa etária entre 18 a 60 anos, divididos em dois grupos:

Desenvolvedores compostos por designers e/ou ilustradores que atuam no mercado profissional e Especialista formado por pesquisadores e/ou professores universitários que lecionem no curso de Design de instituições públicas e/ou privadas. O estudo será composto por 30 participantes ao todo separados em dois grupos com 15 pessoas cada.

Cada participante receberá uma explicação inicialmente, por meio do exemplo já preenchido pelo próprio pesquisador, com a explicação de alguns elementos presentes nas ilustrações procedimentais, incluindo as nomenclaturas abordadas pelo modelo descritivo. Optou-se por a aplicar a explicação dessa maneira, a fim de realizar uma padronização do exemplo da representação para todos os participantes de ambos os grupos. Entretanto esse esclarecimento, não se limitou apenas para o início da pesquisa, estendendo inclusive ao decorrer do procedimento a consulta do participante ao pesquisador a qualquer momento para o esclarecimento de dúvidas em relação ao modelo descritivo no decorrer da pesquisa, de modo que não influencie nas respostas dos participantes.

Após a explicação do modelo descritivo presente no protocolo, inicia-se a pesquisa com a utilização do modelo pelos participantes, a partir da entrega de 10 (dez) modelo descritivo para estilo de ilustração juntamente com a amostra de 10 (dez) ilustrações procedimentais presentes nas bulas de medicamentos.

\section{Resultados}

Os resultados alcançados com o estudo mostram-se bem satisfatórios, com o levantamento inicial das bulas de medicamentos constatou o volume de 277 bulas com ilustrações instrucionais, das quais serão selecionadas para a avaliação dos participantes, para aplicação do modelo descritivo, assim como os resultados alcançados. A seguir, detalharemos cada etapa que compõem o estudo: a) seleção da amostra para aplicação com os participantes; b) Modelo descritivo para estilo de ilustração e c) Validação do modelo descritivo para estilo de ilustração com os participantes.

\subsection{Seleção da amostra para aplicação aos participantes}

A composição da amostra baseou-se na coleta das bulas com ilustrações instrucionais, contidas no bulário eletrônico, contudo a fim de delimitar a validação do modelo descritivo estabeleceu a quantidade de 10 (dez) bulas para aplicação aos participantes. Optou-se em manter a mesma disposição da sequência das ilustrações (e.g. horizontal e/ou vertical) das bulas originais.

A escolha dos estilos visuais das bulas que serão compostas a amostra se desenvolve por meio da variação visual da representação (FUSSELL \& HAALAND, 1978; COOK, 1980; DWYER \& PARKHURST, 1982; SPINILLO, 2001) e a abstração da imagem em si (MCCLOUD, 1995), entretanto observa-se que tal variação ocorre por diferentes fatores, como o nível de detalhes aplicado às SPPs e as técnicas empregada para a sua representação (DONDIS, 1997).

Afim de auxiliar os participantes da pesquisa para o que está sendo representado por meio de imagens, optou-se por manter a posologia da medicação, apresentando-a no canto esquerdo da página, conforme demonstra a Figura 2. Com o objetivo de se manter uma unidade nas representações instrucionais utilizadas na amostra, optou-se em organizalas no formato A4 $(21 \times 29,7 \mathrm{~cm})$ com a impressão coloridas sem brilho em papel sulfite 120 gramas, para cada procedimentos ilustrado das bulas de medicamentos. 


\section{CIDI 2017

Figura 2 - Amostra de número 2 e 3 - Fonte: Elaboração do autor com base em ZOTEON PÓ, 2015 E a direita FLIXOTIDE, 2015.
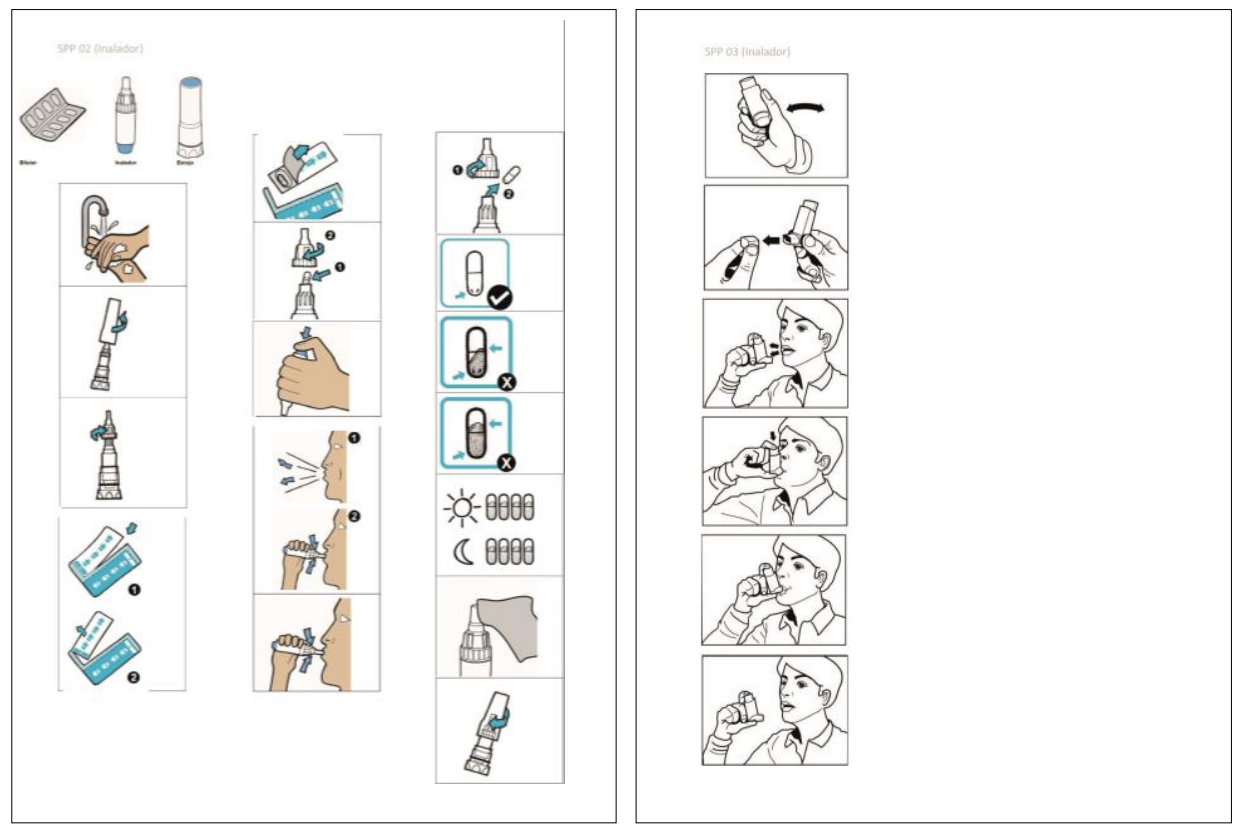

\subsection{Modelo descritivo para estilo de ilustração}

O desenvolvimento do modelo descritivo para estilo de ilustração baseou-se no formato dos modelos Spinillo (2001) e Ashwin (1979). Assim a estrutura para a construção do modelo descritivo buscou abordar as Variáveis do estilo configurado na própria concepção da ilustração, através da forma ou das técnicas empregadas na sua concepção e os

Elementos de representação na SPP como os meios aplicados as ilustrações, mas que não se enquadra ao estilo de ilustração, considerando como um acréscimo e/ou acessórios à imagem.

As categorias empregadas para as Variáveis do estilo de ilustração são as mesmas utilizadas por Ashwin (1979) por meio da sua disposição entre polos opostos, composta por: Cinética, Consistência, Enquadramento, Gama e Proxêmica. Entretanto para a categoria Naturalismo e Não-Naturalista, optou-se pela nomenclatura Realismo, com a variação dos seus polos entre a abstração e o realista na representação, semelhante a escala de representação abordada por McCloud (1995) para as histórias em quadrinhos.

A segunda categoria responsável pelos Elementos de representação em SPPs, fundamentado com base no modelo de Spinillo (2001) em que a autora classifica como: Elementos de Ênfase (cores e formas) e Elementos Simbólicos (barra diagonal e seta). Contudo foram acrescentados outros tipos de variáveis, classificadas como hipérbole para a categoria de ênfase e simbólico para os elementos Xis $(X)$ e Tick $(\checkmark)$.

A elaboração da primeira versão do modelo descritivo para o estilo de ilustração em SPPs, utilizou-se da escala likert para realizar o seu julgamento de valores entre as variáveis gráficas. O Quadro 3, apresenta como foram organizadas essas categorias, assim como as variações dos seus elementos,

Observa que o modelo descritivo para o estilo de ilustração em SPP, também fez o acréscimo de outras categorias como: Ação - abordando expressões visuais que demonstre movimento a ilustração, pelo uso de setas, linhas e múltiplos (e.g sobreposição de imagens). Assim como a presença do indivíduo na representação, como outra categoria acrescentada ao modelo, classificada como participantes distribuídas em três tipos distintos: Agente (o que faz a ação), Objeto (produto que é representado) e Beneficiário (quem recebe a ação representada). 


\section{CIDI 2017

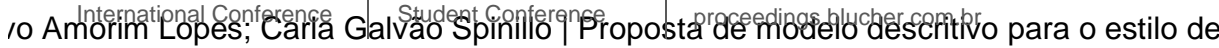
ilustração em Sequência Pictórica de Procedimento em bulas de medicamentos | 87

Quadro 3 - Primeiro modelo descritivo para estilo de ilustração em SPPs. Fonte: Elaborado pelo autor.

\section{Categorias}

Variação de Polos

\begin{tabular}{|c|c|c|c|c|c|c|c|c|}
\hline \multirow{6}{*}{ 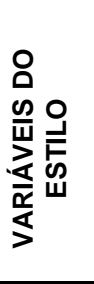 } & Cinética & Estática & 1 & 2 & 3 & 4 & 5 & Dinâmica \\
\hline & Consistência & Homogênea & 1 & 2 & 3 & 4 & 5 & Heterogênea \\
\hline & Enquadramento & Conjuntiva & 1 & 2 & 3 & 4 & 5 & Disjuntiva \\
\hline & Gama & Restritiva & 1 & 2 & 3 & 4 & 5 & Expandida \\
\hline & Proxêmica & Próxima & 1 & 2 & 3 & 4 & 5 & Distante \\
\hline & Realismo & Abstrato & 1 & 2 & 3 & 4 & 5 & Realismo \\
\hline \multirow{4}{*}{ 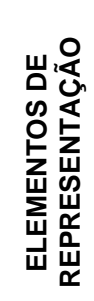 } & Categorias & \multicolumn{6}{|c|}{ Tipos } & Outros \\
\hline & Ênfase & Cor & \multicolumn{2}{|c|}{ Hipérbole } & & \multicolumn{2}{|c|}{ Forma } & \\
\hline & Participante & Agente & \multicolumn{2}{|c|}{ Objeto } & & \multicolumn{2}{|c|}{ Beneficiário } & \\
\hline & Simbólico & Xis $(\mathbf{X})$ & \multicolumn{2}{|c|}{ Barra $(/)$} & & \multicolumn{2}{|c|}{ Tick $(\boldsymbol{})$} & \\
\hline
\end{tabular}

Com o propósito de verificar o desempenho da primeira versão do modelo, realizou a avaliação em 10 (dez) SPPs escolhidas de forma aleatórias com base na coleta do estudo analítico. De modo que os resultados alcançados nessa descrição foram verificados, mesmo não sendo o objetivo da análise, por buscar apenas a descrição qualitativa com o uso do modelo descritivo em SPPs. Assim os resultados alcançados com está primeira averiguação observou os seguintes pontos:

O primeiro com relação à utilização da escala Likert, por não desempenhar uma avaliação satisfatória para descrição do estilo de ilustração, por realizar a observação de apenas o conjunto ilustrado, não possibilitando a descrição individual das imagens nos quadros que compõem a sequência instrucional.

O segundo ponto verificado é ausência do espaço aberto destinado para anotações e observações das SPPs através do modelo descritivo, aplicado as variáveis do estilo de ilustração, conforme estão presentes nas categorias dos elementos de representação, destinadas a está função.

Para o desenvolvimento da segunda versão do modelo descritivo para estilo de ilustração buscou apenas realização de pequenos ajustes com a primeira versão do modelo, conforme descrito anteriormente. Está segunda versão, optou pela exclusão da escala Likert, utilizada para descrever as variáveis do estilo de ilustração, alterando dessa forma para a realização de uma análise individual dos quadros representados nas SPPs. Outro ponto alterado é a inclusão de uma área destinada a observação da imagem, com informações e/ou comentários relacionados à descrição do estilo, conforme apresenta-se de forma detalhada no Quadro 4. 


\section{CIDI 2017

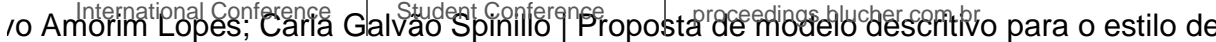
ilustração em Sequência Pictórica de Procedimento em bulas de medicamentos | 88

Quadro 4 - Segunda versão do modelo descritivo para estilo de ilustração em SPPs. Fonte: Elaborado pelo autor

\begin{tabular}{|c|c|c|c|c|}
\hline & Variáveis & Tipos & Quantidade & Observação \\
\hline \multirow{11}{*}{ 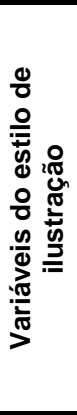 } & Consistência & \multicolumn{2}{|c|}{$\begin{array}{l}\text { Homogênea } \\
\text { Heterogênea } \\
\end{array}$} & \\
\hline & \multirow{2}{*}{ Cinética } & Estática & & \\
\hline & & Dinâmica & & \\
\hline & \multirow{2}{*}{ Enquadramento } & Conjuntivo & & \\
\hline & & Disjuntivo & & \\
\hline & \multirow{2}{*}{ Gama } & Restrita & & \\
\hline & & Expandida & & \\
\hline & \multirow{2}{*}{ Proxêmica } & Próximo & & \\
\hline & & Distante & & \\
\hline & \multirow{2}{*}{ Realismo } & Abstrato & & \\
\hline & & Realista & & \\
\hline & & & Elementos & Observação \\
\hline \multirow{12}{*}{ 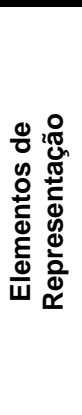 } & \multirow{3}{*}{ Participante } & Agente & & \\
\hline & & Objeto & & \\
\hline & & Beneficiário & & \\
\hline & \multirow{3}{*}{ Ação } & Seta & & \\
\hline & & Linha & & \\
\hline & & Múltiplas & & \\
\hline & \multirow{3}{*}{ Ênfase } & Cor & & \\
\hline & & Hipérbole & & \\
\hline & & Forma & & \\
\hline & \multirow{3}{*}{$\begin{array}{l}\text { Símbolo / } \\
\text { convenção }\end{array}$} & Xis $(X)$ & & \\
\hline & & Barra (/) & & \\
\hline & & Tick $(\checkmark)$ & & \\
\hline
\end{tabular}

A segunda versão do modelo descritivo, optou-se por as mesmas características da primeira versão, realizando apenas algumas alterações em relação a disposição das categorias avaliadas, ao invés do formato horizontal para o vertical. Outro ponto alterado é a inclusão de uma coluna única com a função de descrever a quantidade de quadros das SPPs, que são distribuídos entre os polos opostos para as variáveis do estilo de ilustração. Entretanto para a categoria Consistência não se faz a classificação entre os polos por meio do número de quadros, por realizar a descrição da unidade da variável, através do posicionamento único entre o campo homogêneo e ou heterogênea.

A segunda parte do modelo descritivo, destinada aos elementos de representação, busca apenas indicar a presença ou ausência dos elementos visuais nas SPPs, também alterando sua estrutura para o formato vertical, assim como seus elementos, afim de manter uma unidade no modelo descritivo.

Com o objetivo de verificar o desempenho dessa segunda versão do modelo, optou-se pela avaliação em 10 (dez) SPPs, sendo as mesmas que compõem a amostra para a validação com os participantes da pesquisa, além de avaliar a funcionalidade do modelo descritivo, também será utilizado como base para se verificar os resultados com participantes.

\subsection{Validação do modelo descritivo para estilo de ilustração com os participantes}

A validação do estudo realizou-se individualmente com ambos os grupos de participantes (Desenvolvedores e Especialistas), contudo como o objetivo de padronizar a explicação aos participantes utilizou-se o modelo da SPP pré-definido (Figura 3), a fim de demonstrar as variáveis descritivas do estilo e os seus elementos visuais que compõem as instruções, com a apresentação em maior ênfase e a localização de suas variável e seus elementos visual, seta, agente, cinética com a presença do elemento múltiplo, linhas/espaço e a variação de cores e formas, apresentando algumas das características que são abordadas pelo modelo descritivo. 


\section{CIDI 2017}

Figura 3 - SPP explicativa do modelo. (Elaboração do autor com base na Bula CLENIL COMPOSTION HFA, 2014. p.6).

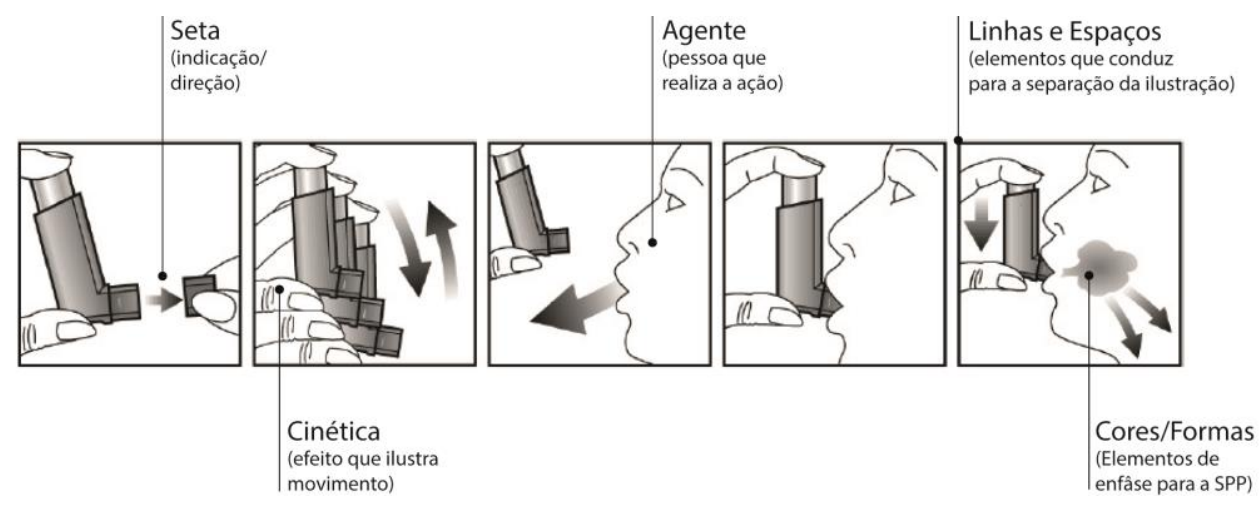

\subsubsection{Perfil dos participantes}

A composição do perfil dos participantes foi realizada com 30 voluntários, divididos em dois grupos iguais de 15 participantes cada, classificados como Desenvolvedores e Especialistas.

Os perfis dos participantes em ambos os grupos alcançaram resultados semelhantes, com uma amostra mista entre homens e mulheres que compõem o estudo: Desenvolvedores 8 mulheres e 7 homens, e para os Especialistas 9 homens e 6 mulheres. A faixa etária entre os dois grupos com a maior evidência para os Desenvolvedores, idade 18 a 30 anos e para os Especialistas variando entre 26 a 40 anos.

A escolaridade é outro fator observado no perfil dos participantes, verificando que ambos os grupos tinham concluído ou cursavam uma Pós-Graduação Stricto Sensu, nota que o grupo de Especialistas, obteve uma maior ênfase para o Mestrado Completo, que ao confrontar com as informações profissionais constituem de docentes universitários. Para os desenvolvedores a escolaridade prevaleceu o Mestrado Incompleto, e atuação profissional como Designers com maior incidência. O período que desempenham a função foi outro fator avaliado, demonstrando um tempo maior para os Especialistas com uma média de 6 a 15 anos enquanto para os Desenvolvedores é de 1 a 10 anos de experiência com a função.

\subsubsection{Resultados do modelo descritivo: Desenvolvedores X Especialistas}

A construção da discussão entre grupos de participantes faz uma avaliação do conjunto com as variáveis separadas por categorias, com objetivo de melhor apresentar seus resultados. A fim de sintetizar tais resultados alcançados, apresentaremos a discussão entre grupos (Desenvolvedores e Especialistas), e os seus principais comentários expostos sobre o modelo, elaborado com as variáveis do estilo de ilustração seguido pelos elementos de representação.

Na categoria Consistência, com o mesmo resultado em ambos os grupos para homogêneo, sendo o principal aspecto de influência na ilustração por meio da variação de traço, linhas e contornos considerando como uma característica importante para se manter uma unidade na Sequência Pictórica de Procedimento.

A Cinética, a segunda variável aborda pelo modelo, destaca-se por ambos os grupos para a categoria Dinâmica, classificação por elencar o movimento a representação, normalmente associadas ao uso dos elementos visuais, mencionados pelos dois grupos como o uso da seta e linhas, para configurar o movimento na representação. Contudo apenas os Desenvolvedores elencaram para o recurso de "llustração Narrativa" com o objetivo de gerar movimento no decorrer da sua própria sequência por meio dos quadros ilustrados.

Para o Enquadramento, a categoria que evidência em ambos os grupos a disjuntiva, classificada pelos desenvolvedores como a forma de descrever o fundo presente na representação, classificada como a principal variação na ilustração, destacando o fundo 
em degrade. Entretanto os Especialistas determinam como os elementos que resaltam a imagem, ou seja, que dão um maior destaque, não se limitando unicamente pelo fundo que caracteriza a ilustração procedimental.

A quarta variável abordada é a Gama, classificada por ambos os grupos de participantes como expandida, por configurar a ampliação dos seus elementos que a compõem, alterando entre traços, contornos, hachuras, texturas, aplicação de cores e uso de degrade, de modo a compor tais características, semelhantes à categoria da variável consistência descrita anteriormente. Para os especialistas, a gama constitui das alternâncias técnicas empregadas à representação, fator que diferencia o estilo, assim como um acréscimo dos elementos a ilustração em si, como setas e/ou textos, expandindo os seus significados.

A variável Proxêmica é classificada por ambos os grupos como a categoria próxima, identificada pela escala do objeto representado. Para os Desenvolvedores está relação se constrói, com base no próprio agente representado nas SPPs, realizando a sua aproximação dos quadros, a partir da alteração em seu tamanho ilustrado. Porém, os especialistas afirmam que essa variação ocorre decorrente dos detalhes presentes na imagem, assim como o recurso de close, com o objetivo de detalhar a imagem, utilizado com frequências no cinema, por meio da aproximação da câmera.

A última variável do estilo de ilustração o Realismo, obteve os mesmos resultados pelos dois grupos de participantes, para a categoria realista, suas características fazem comparação com as informações dos objetos real, além de associar o participante ao beneficiário, desenvolvendo as mesmas funções dentro da SPP. No entanto para os especialistas classificam está variável pela redução dos elementos representados como: texturas e volume, transformando a ilustração mais abstrata.

Os elementos de representação são distribuídos em quatro categorias: Participantes, Ação, Ênfase e Símbolo/Convenções. O primeiro elemento classificado como Participantes, resalta a presença do agente e o objeto na SPPs, abordado por ambos os grupos de participantes como o "agente é o beneficiário" por desenvolverem a mesma função na ilustração, quando aplicado a presença do beneficiário no procedimento.

Como forma de detalhar a Ação, é a opção presente em ambos os grupos e em toda a amostra verificada, destacou-se o recurso de seta, para a demonstração de direção, movimento e sentido, configurando como o elemento mais mencionados entre os participantes para as SPPs. Assim como a Linha, classificada como elementos de ação, para expressar o ar/sopro, configurando como os elementos de maior dificuldade entre os participantes e em ambos os grupos, já que algumas instruções as linhas não representavam uma ação, e sim outras informações (e.g. indicação, inventarial).

A Ênfase destaca-se em ambos os grupos para as categorias cor e forma, a primeira aplicada apenas aos elementos visuais (e.g. setas, molduras, box, xis, entre outros), e a segunda à forma, faz a configuração em diversos modos como molduras, variações de linhas (e.g. indicação, inventarial, posicionamento), incluindo a seta considerada pelos especialistas como uma forma de representação. Assim outro ponto aborda por ambos os grupos é a inclusão do Zoom e a vista seccionada a ilustração, para o interior da categoria hipérbole além dessas características, outro modo abordado pelos grupos de especialistas, é o uso de expressões exageradas nas ilustrações, representado na boca para a para demonstração da respiração do personagem, de modo a enfatizar a ação na SPP.

A última variável apresentada classificada como símbolos e convenções, foram as que alcançaram os melhores desempenhos de entendimento para os seus significados dentro da SPP, destacando para o recurso do Xis entre os Desenvolvedores e os elementos $X$ is e a barra entre os especialistas. As definições das variáveis foram bem unanimes em ambos os grupos, definindo o xis como forma de restrição, proibição ou de erro e o tick como elemento de indicação de acerto, contudo para a barra foi a única variável que distorceu o seu significado, configurando como um elemento de divisão de quadro e/ou elementos presentes na SPP, e não como uma representação de advertência ou proibição, como é destinado o seu significado inicialmente. 


\section{Considerações finais}

O presente estudo propôs validar o modelo descritivo para estilo de ilustração em Sequência Pictórica de Procedimento em bulas de medicamentos, seja com o preparo e/ou aplicação da própria medicação. A validação conduzida por meio do modelo buscou verificar a ilustração procedimental em duas etapas, a primeira aplicada as variáveis do estilo e a segunda pelos elementos de representação.

Inicialmente o modelo aplicado as variáveis do estilo de ilustração fizeram a descrição por quadros separados das SPPs verificadas, entretanto observou que está distribuição da quantidade de quadros entre as categorias, não se alcançou diferenças entre os quadros isolados da sequência instrucional completa, de modo que tal abordagem foi vista por muitos participantes como confuso o seu preenchimento no modelo para a descrição das SPPs que compõem a amostra. Nessa parte do modelo outros questionamentos levantados pelos participantes foram em relação às nomenclaturas abordadas pelo modelo dificultando a compreensão e o seu entendimento, destacando entre eles para: gama, enquadramento e proxêmica.

Para o variável enquadramento, verificada em primeiro plano como a disposição dos elementos que compõem a ilustração, consistindo da restrição ou expansão do fundo da imagem, mas também a forma que a imagem se distribui dentro do seu campo delimitador, seja Box, quadro, tabela entre outro recurso presentes nos quadros das SPPs. A nomenclatura utilizada para descrição das variáveis, conjuntiva e disjuntiva, destaca como difícil o entendimento e assim como os seus significados para realizar a distinção.

A gama, assim como o enquadramento encontrou-se maiores dificuldades para a categorias Restrita e Expandida. Contudo essa variável enquadra nas possibilidades sintáticas da imagem, como expandida, classificada pelo uso de hachuras, traços, cores, linhas e elementos visuais e restritos com a limitação dessas características, entretanto observa que a ampliação da gama, torna a ilustração mais próxima de uma imagem real, detalhada no campo destinado a variável realista.

A proxêmica configura como a relação do objeto com a aproximação ou distanciamento do leitor, através da variação da escala no objeto. Algumas características podem ser consideradas como o posicionamento do ângulo que é representado, destacando-o para o estilo fotográfico que faz o uso de técnicas de posicionamento de câmeras, através da linguagem cinematográfica não se limitando apenas a fotografia, expandindo também a ilustração no geral, com o uso das técnicas de Close e Zoom para aproximar do objeto ou enquadramento dos detalhes nas SPPs.

A validação do modelo possibilitou verificar a exclusão do item quantidade, realizando a avaliação de todo o procedimento, e a alterações das nomenclaturas a fim de facilitar o seu entendimento destacando-o a Gama com a alteração para Técnica e o Enquadramento como Plano, e realizou a opção da retirada da variável Proxêmica do modelo.

As categorias dos elementos de representação, em especial as variáveis ação e ênfase, a primeira por acrescentar outros tipos de elementos para demonstração de movimento para a ilustração como (aspas, linhas e seta de ação), e a segunda variável ênfase na SPPs optou-se pela retirada da categoria forma, considerada como uma nomenclatura muito abrangente dificultando o posicionamento de alguns elementos, e com objetivo de delimitar as variáveis optou-se por distribuir os elementos mais reocorrentes na SPPs como: Círculo, Linhas, Quadrados, Setas e o Zoom/Close, sendo este último item, elencando pelos próprios participantes como forma de ênfase no objeto representado. Contudo vale resaltar para o elemento linha que são atribuídos os mais diversos tipos de variações: Linhas da imagem (inventarial); Linha de Posicionamento; Linha de Legendas; Linhas de Indicação, de modo a que para todos os tipos são atribuídos significados diferentes.

A categoria responsável pelo uso de convenções e símbolos é a variável alcançou os melhores desempenhos em relação ao seu significado que se aplica a ilustração, conforme detalha os próprios participantes como restrição e feedback na sua utilização. A barra é a única classificada com outro significado, ao invés de sinalizar proibição e/ou 
advertências, é atribuída como um elemento apenas para divisão de quadros dentro da própria SPPs.

O desempenho do modelo para descrever a ilustração é alcançado, assim como a forma que é detalhada os quadros representados, porém observa-se que a categoria quantidade, não é determinante para descrever o estilo, contudo o campo de observação em que os participantes detalhavam a ilustração ou indicavam os quadros da ilustração que devesse resaltar, ou apresente informações complementares, alcançaram os melhores resultados quando comparados a categorias de quantidade. Da mesma maneira, o preenchimento do modelo destinado aos elementos de representação, ausentando para a descrição da quantidade presente nos quadros, tornando mais simples o seu preenchimento pelos participantes.

\section{Agradecimento}

Agradeço a CAPES pelo apoio financeiro na concessão de bolsa de estudos e à minha orientadora, Dra. Carla Galvão Spinillo por todo apoio, orientação e dedicação, assim como todos os participantes do estudo por tornar possível está investigação.

\section{Referências}

ASHWIN, C. The ingredients of style in contemporary illustration: a case study. Information design journal, v. 1, n. 1, 1979. p. 51-67

ANZOLCH, R. 2009:16. Geometria do estilo: genealogia da noção da arquitetura. Tese (Doutorado em Arquitetura). Universidade Federal do Rio Grande do Sul. Porto Alegre, 2009.

BRASIL. Ministério da Saúde. Agência Nacional de Vigilância Sanitária. Resolução n ${ }^{\circ} 140$, de 29 de maio de 2003. Diário Oficial da União, Brasília, DF, 24 set. 2003. Seção 1, p. 53-54.

BRASIL. Ministério da Saúde. Agência Nacional de Vigilância Sanitária. Resolução $n^{\circ} 47,9$ de setembro de 2009. Diário Oficial da União, Brasília, DF, 9 set. 2009b. Seção 1, p. 40

CLENIL COMPOSITUM HFA. Responsável técnica C. M. H. Nakazaki. Santana de Parnaíba: Chiesi Farmacêutica Ltda., 2014. Bula de remédio.

COOK, B. Picture Communication Research Findings from Papua New Guinea, Educational Broadcasting International, vol. 13, no. 2 (1980), pp. 78-83.

DA SILVA, C. H.; SPINILLO, C. G. Dificuldades e estratégias no uso de múltiplos medicamentos por idosos no contexto do design da informação. Estudos em Design, $v$. 24, n. 3, 2016.

DONDIS, D. A. Sintaxe da linguagem visual. 2.ed. São Paulo: Martins Fontes, 1997.

DWYER, F. M.; PARKHURST; P. E. A Multifactor Analysis of the Instructional Effectiveness of Self-Paced Visualized Instruction on Different Educational Objectives. Programmed Learning, v. 19, n. 2, p. 108-118, 1982

FLIXOTIDE. Responsável técnico: Responsável técnico: Edinilson da Silva Oliveira. Rio Janeiro: GlaxoSmithKline Brasil Ltda., 2015. Bula de remédio.

FUJITA, P. T. L.; SPINILLO, C. G. A importância da informação no processo de aquisição e uso de medicamentos no Brasil. In: 80 Congresso Internacional de Ergonomia e Usabilidade e Design de Interfaces e Interao Humano-Computador, 2008, São Luís, MA. Anais do 8 USIHC. São Luiz, MA: UFMA, 2008.

FUJITA, P. T. L.; MACHADO, C. J. S.; DE OLIVEIRA TEIXEIRA, M. A bula de medicamentos e a regulação de suas configurações em termos de forma e conteúdo no Brasil. Saúde e Sociedade, v. 23, n. 1, p. 277-292, 2014

FUSSELL, D.; HAALAND, A. Communicating with Pictures in Nepal: Results of Practical Study Used in Visual Education. Educational Broadcasting International, v. 11, n. 1, p. 2531, 1978. 
GOMBRICH, E. H. Arte e ilusão: um estudo da psicologia da representação pictórica. São Paulo: Martins Fontes, 1995, 384p.

GONÇALVES, S. A.; MELO, G. D.; TOKARSKI, M. H. L., BARBOSA-BRANCO, A. Bulas de medicamentos como instrumento de informação técnico-científica. Revista de Saúde Pública, v. 36, n. 1, p. 33-39, 2002.

HAMMERSCHMIDT, C.; SPINILLO, C. G. Informações sobre medicamentos em dispositivos móveis: Análise da articulação tipográfica no aplicativo MedSUS. Blucher Design Proceedings, v. 2, n. 2, p. 753-765, 2015.

HARADA, F. J. B.; SCHOR, P. Design centrado no humano para a compreensão problemas de pacientes idosos com deficiência visual. Human Factors in Design, v. 5, n. 9, p. 018037, 2016.

LAKATOS; E. M. MARCONI, M. A. Fundamentos de metodologia cientifica. 3. ed. São Paulo: Atlas, 1996.

MCCLOUD, S. Desvendando os quadrinhos. São Paulo: M. Books, 1995.

PARANÁ. Secretaria de Estado da Saúde. Medicamentos manipulados terão bulas padronizadas no Paraná. 2013a. Disponível em: <http://goo.gl/jTwTZ4> Acesso 15 de abril de 2016.

PARANÁ. Secretaria de Estado da Saúde. Resolução SESA no 62. 2013b. Disponível em: <http://goo.gl/46PkLl>. Acesso 15 de abril de 2016.

REDIG, J. Não há cidadania sem informação, nem informação sem design. InfoDesign, Pernambuco, v. 1, 2004.

SCARIOTI, C. A.; DE ARAÚJO GOMES, Í. M. Design da informação para documentos públicos: aspectos e considerações relevantes para o desenvolvimento de bulas de medicamentos. $4^{\circ}$ Congresso Internacional de Design da Informação $3^{\circ}$ InfoDesign Brasil. Rio de Janeiro. 2009.

SPINILLO, C. G. Instruções visuais: algumas considerações e diretrizes para sequencias pictóricas de procedimentos. Estudos em Design. Volume 9, n 3, Rio de Janeiro, 2001.

SPINILLO C.G; PADOVANI, S.; MIRANDA, F.; FUJITA, P. T. L. Instruções visuais em bulas de medicamentos no Brasil: um estudo analítico sobre a representação pictórica da informação. In: $3^{\circ}$ Congresso Internacional de Design da Informação, 2007, Curitiba.

SPINILLO; C. G. FUJITA, P. T. L. Design da informação em bulas de medicamento: análise e classificação da estrutura e apresentação gráfica de seu conteúdo textual. Rev Bras Desig Inform, v. 5, n. 3, p. 1-12, 2008.

SPINILLO, C. G.; PADOVANI, S.; LANZONI, C. Ergonomia informacional em bulas de medicamentos e na tarefa de uso: um estudo sobre fármaco em suspensão oral. Revista Ação Ergonômica, v. 5, n. 1, 2011.

SPINILLO, C. G.; WAARDE, K. Design de instruções visuais em bulas de medicamentos numa perspectiva internacional: estudo comparativo entre Brasil e Comunidade Europeia. In: 5ํㅡㄹ Congresso Internacional de Design da Informação. 2011.

TWYMAN, M. L. Using pictorial language: a discussion of the dimensions. Designing usable text, p. 245-312, 1985.

ZOTEON PÓ. Responsável técnica C. M. H. Nakazaki. São Paulo: Novaris Biociências S.A., 2015. Bula de remédio.

\section{Sobre os autores}

Luiz Gustavo Amorim Lopes, Mestre em Design, Universidade Federal do Paraná (UFPR), Brasil, <gustavo.amorim.1904@gmail.com>.

Carla Galvão Spinillo; PhD em Design e Docente da Universidade Federal do Paraná (UFPR), Brazil <cgspin@gmail.com>. 